\title{
Editorial
}

\section{Exercise Physiology, Cognitive Function, and Physiologic Alterations in Extreme Conditions 2016}

\author{
Ellen L. Glickman, ${ }^{1}$ Edward J. Ryan, ${ }^{2}$ and David Bellar ${ }^{3}$ \\ ${ }^{1}$ School of Health Science, Exercise Physiology, Kent State University, Kent, OH 44242, USA \\ ${ }^{2}$ Exercise Science, Chatham University, Pittsburgh, PA 15232, USA \\ ${ }^{3}$ School of Kinesiology, University of Louisiana at Lafayette, Lafayette, LA 70506, USA \\ Correspondence should be addressed to Ellen L. Glickman; eglickma@kent.edu
}

Received 30 October 2016; Accepted 31 October 2016

Copyright ( 92016 Ellen L. Glickman et al. This is an open access article distributed under the Creative Commons Attribution License, which permits unrestricted use, distribution, and reproduction in any medium, provided the original work is properly cited.

The field of exercise physiology is a broad field of inquiry that focuses on the total physiological responses of humans to stressors such as exercise or the environment. While it is well known that extremes environments impose unique stressors on humans that manifest profound physiological effects, it remains an area that is underreported upon in the literature. It should be noted that it is often through the study of physiological extremes that the scientific community gains greater insight into the capacity of the human organism for physiological function.

Furthermore, it has been shown that extreme environments have a detrimental effect on cognitive function and also result in elevated inflammation and changes in metabolism. Though not commonly experienced by all people, there are instances either occupationally or recreationally where people not only are exposed to extreme environments, but also are required to compound the stress through exercise. The resulting combination can manifest even greater physiological responses, which require investigation.

The editors invited contributions for this special edition that represented current and timely expansion of knowledge in the areas of environmental and exercise physiology in extreme conditions. It is of utmost importance that inquiry in this area continues so that a more comprehensive understanding of the human physiological response to unique environments can be developed and methods for coping with these environmental stressors improved.
Ellen L. Glickman Edward J. Ryan David Bellar 

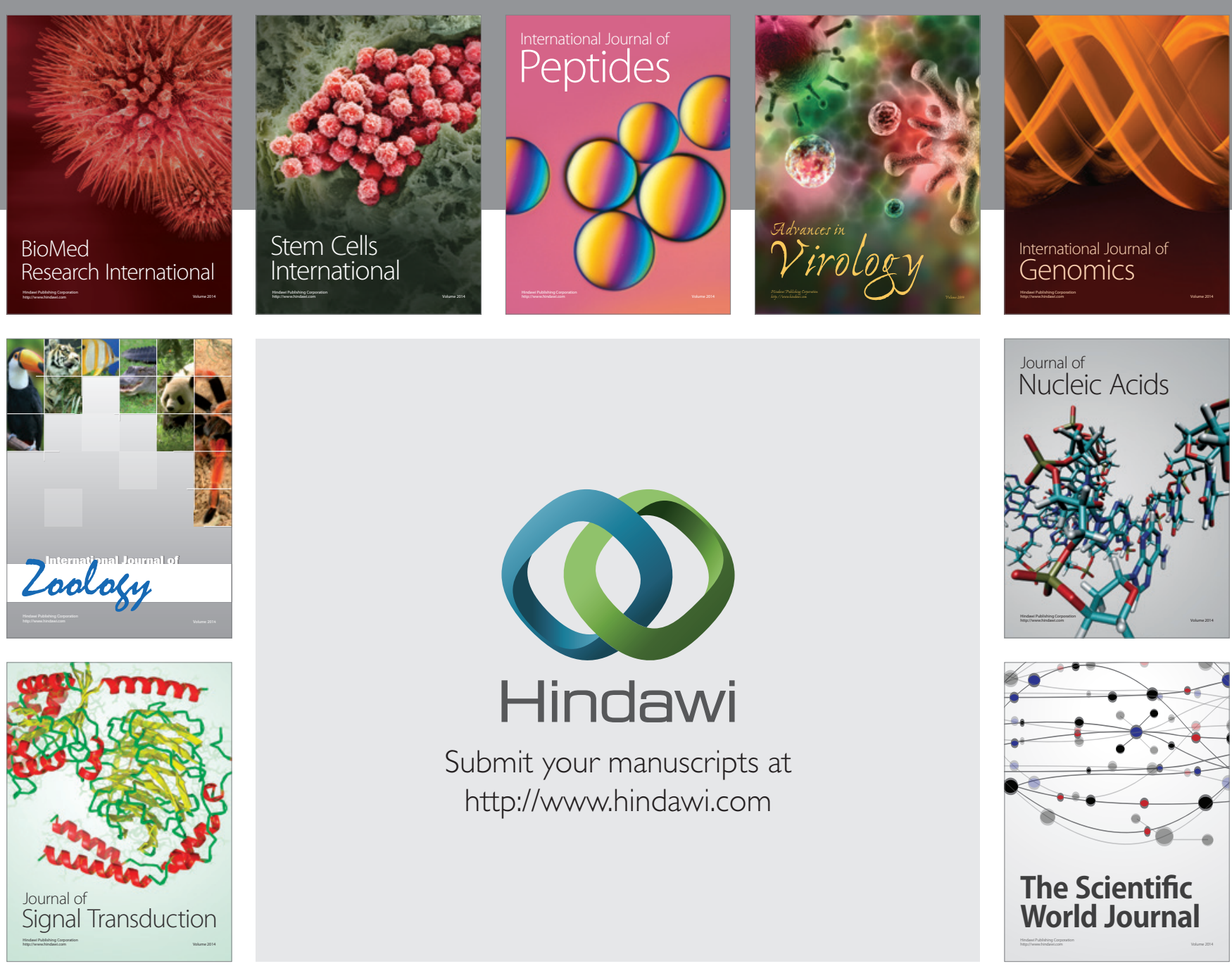

Submit your manuscripts at

http://www.hindawi.com
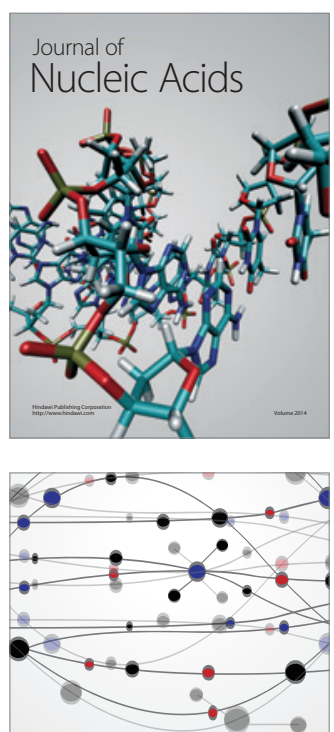

The Scientific World Journal
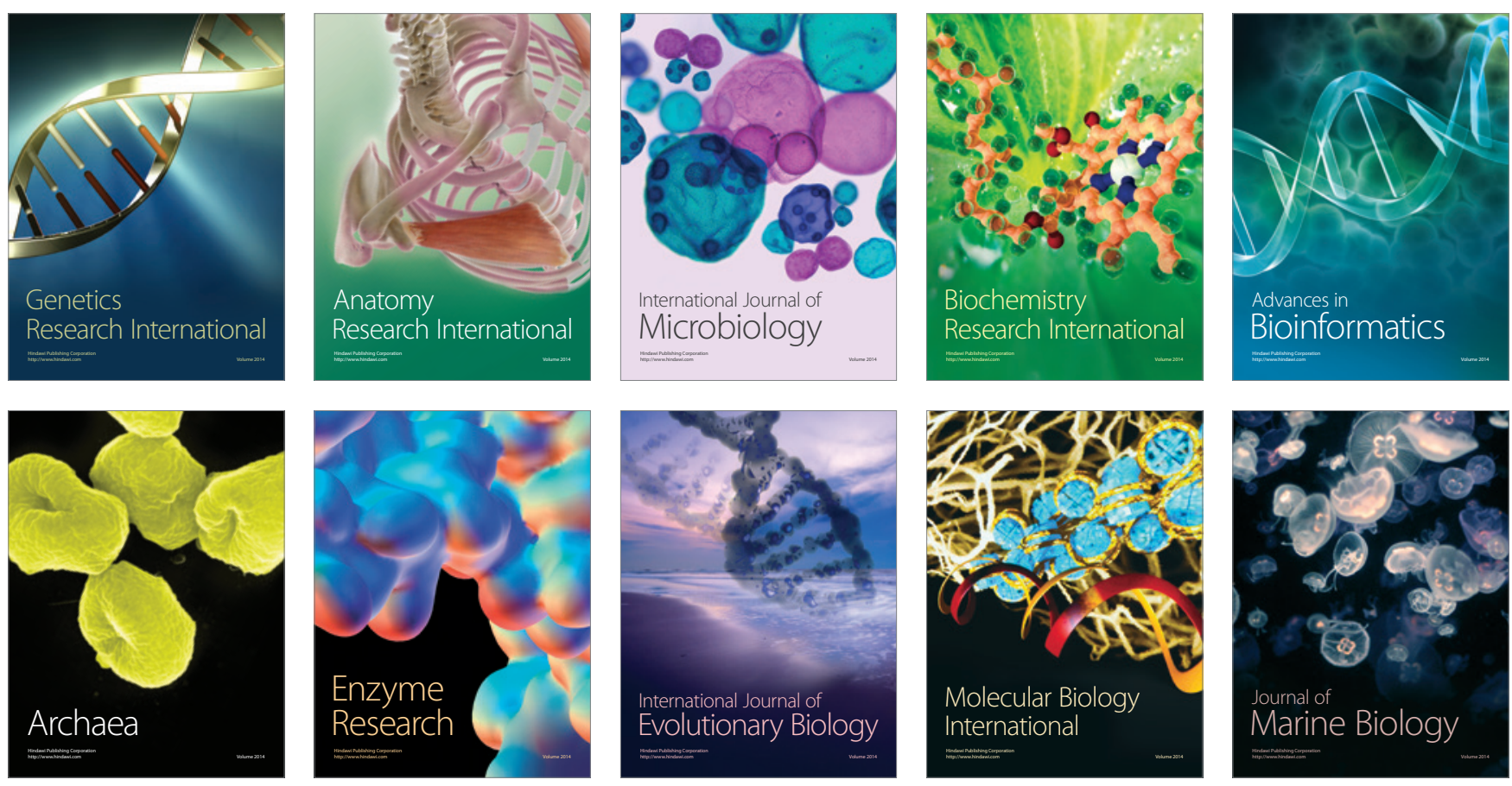